\title{
Improved Assessment of Interference Limits in Cellular Radio Performance
}

\author{
Ramjee Prasad, Senior Member, IEEE, and Adriaan Kegel
}

\begin{abstract}
Reuse distance and cluster size are evaluated for cellular mobile radio systems. Rayleigh fading, log-normal shadowing, and area mean power inversely proportional to the fourth power of propagation distance are considered, using the technique of Schwartz and Yeh for determining the probability density function of the sum of several log-normal variables in order to obtain the cochannel interference probabilities. These results are used for analyzing the radio spectrum efficiency, taking also traffic intensity into consideration. Different modulation methods, namely, analog FM with 30 - and $12.5-\mathrm{kHz}$ channel spacing, SSB with 5-kHz spacing, and digital modulation with $25-\mathrm{kHz}$ channel spacing, with adequate protection ratios, are compared. An example of the calculation of system bandwidth and cluster size is presented using this procedure.
\end{abstract}

\section{INTRODUCTION}

I MPROVED assessment of cochannel interference in cellular systems in order to evaluate the system performance has enjoyed a high priority in mobile radio research during the last decade, e.g., [1]-[10]. Generally, three propagation aspects are considered: random (fast) multipath fading, random (slow) shadowing, and the deterministic UHF groundwave path loss. A difficult problem arises in developing the corresponding interference model for several fading radio signals.

The probability density function (pdf) of the sum of random signals with log-normal probability distribution is required. An approximate pdf was obtained in [1]-[3] using Fenton's method [11], and in [10] using Wilkinson's approach [12]. However, both methods are unfortunately suitable for small signal variance $\left(\sigma^{2}\right)$ only, while typical values of $\sigma$ lie between 6 and $12 \mathrm{~dB}$ in mobile channels. An improved technique for approximating the probability distribution of the sum of several random variables with such high variances, based on a method by Schwartz and Yeh [12], has recently been reviewed [13]. The present paper deals with the assessment of capacity for a mobile radio system, in terms of reuse distance, cluster size, and spectrum efficiency based on the concept of required protection ratio. Capacity is calculated by evaluating the probability of cochannel interference of a system exposed to multipath fading and shadowing, using Schwartz and Yeh's technique [12], [13]. The motivation for this study of mobile $(\mathrm{CW})$ telephony are the findings

Manuscript received September 19, 1989 and February 6, 1990; revised November 20,1989, March 26, 1990, and October 10,1990. This is an expanded version of the work originally presented at the 39 th IEEE Vehicular Technology Conference, San Francisco, CA, May 1989.

The authors are with the Telecommunications and Traffic Control Group, Delft University of Technology, $2600 \mathrm{GA}$ Delft, The Netherlands.

IEEE Log Number 9143053. in recent studies of mobile (bursty) packet systems [14], [15] in which it was reported that Fenton's classical method is not well suited for applications in typical mobile environments with high $\sigma$.

Spectrum efficiency $\left(E_{s}\right)$, expressed in erlangs per megahertz per square kilometers (erlang $/ \mathrm{MHz} / \mathrm{km}^{2}$ ) is defined as the ratio of carried traffic per cell and the product of bandwidth, number of cells per cluster and cell area. Thus $E_{s}$ is an important parameter for assessing the frequency requirements of cellular mobile radio systems.

It is necessary to consider whether several cochannel interference signals add coherently or incoherently. Coherent addition of phasors would be more appropriate, if the random phase terms of the individual interferers hardly vary during the receive period; this would seem an impractical proposition for mobile telephony. Incoherent cumulation of cochannel interferers in the analysis of the system performance of cellular mobile radio telephony is a more realistic assumption. However, coherent cumulation of interferers was assumed in the analysis presented in [1], [13] by considering that $n$ Rayleigh-distributed phasors add up to one Rayleighdistribution phasor, with mean power equal to the sum of local mean interference powers [15], [19]. Therefore, the corresponding pdf for the joint interference power is an exponential distribution. In the case of incoherent cumulation of interferers, with equal mean power, the pdf for the joint interference power would rather be given by the gamma distribution [19]. Studies of the throughput of packet radio in typical mobile channels with multiple incoherent interferers [16] indicate that the coherent interference model gives more optimistic results for the spectrum efficiency than the incoherent model. This result for burst-mode systems is confirmed here for typical continuous-mode mobile radio telephony systems with multipath fading and shadowing.

This paper is organized as follows. Section II formulates the probability of cochannel interference for uncorrelated fading signals. Section III describes the computational results of cochannel interference probability and reuse distance. In Section IV, the effect of cluster size, protection ratio and carried traffic on the spectrum efficiency is discussed. Section $\mathrm{V}$ defines the system bandwidth and computational results are presented for different modulation schemes and parameters. Section VI contains the concluding remarks.

\section{Formulation of Cochannel InTERference Probability}

The cellular telephony concept has been described in numerous papers, e.g., [17]. In order to investigate the reuse 
distance and spectrum efficiency of a cellular system, it is necessary to determine the cochannel interference probability [1]-[10], defined as

$$
F(C I) \triangleq \sum_{n} F(C I \mid n) F_{n}(n) .
$$

Here $F_{n}(n)$ is the probability of $n$ cochannel interferers being active. $F(C I \mid n)$ is the corresponding conditional cochannel interference probability,

$$
F(C I \mid n) \triangleq \operatorname{prob}\left\{p_{d} / p_{n}<\alpha\right\}
$$

where $p_{d}$ is the instantaneous power of the desired signal, $p_{n}$ is the joint interference power from $n$ active channels, and $\alpha$ is the specified cochannel protection ratio.

The (fast) amplitude fluctuation of mobile radio signals is described by the Rayleigh density function. The pdf for the signal amplitude $r_{i}$ of the $i$ th interferer, conditional on its local mean power $p_{0 \mathrm{i}}$, is given by

$$
f_{r i}\left(r_{i} \mid p_{0 i}\right)=\frac{r_{i}}{p_{0 i}} \exp \left(-r_{i}^{2} / 2 p_{0 i}\right) \text {. }
$$

The corresponding pdf for the instantaneous power $p_{i}$ is

$$
f_{p i}\left(p_{i} \mid p_{0 i}\right)=\frac{1}{p_{0 i}} \exp \left(-\frac{p_{i}}{p_{0 i}}\right) \text {. }
$$

The local mean power $p_{0 i}$ is itself a (more slowly varying) stochastic variable with the log-normal pdf

$$
f_{p_{0 i}}\left(p_{0 i}\right)=\frac{1}{\sqrt{ }(2 \pi) \sigma_{i} p_{0 i}} \exp \left\{-\left(\ln p_{0 i}-m_{i}\right)^{2} / 2 \sigma_{i}^{2}\right\} \text {. }
$$

Here, $\sigma_{i}^{2}$ and $\xi_{i}=\exp \left(m_{i}\right)$ are the logarithmic variance and the area mean power of the interfering signal, respectively.

The amplitude, $r_{d}$, instantaneous power, $p_{d}$, and local mean, $p_{0 d}$, of the desired signal can also be described by density functions of the forms (3)-(5), respectively, with the following substitutions:

$$
\begin{aligned}
r_{i} & \rightarrow r_{d} \\
p_{i} & \rightarrow p_{d} \\
p_{0 i} & \rightarrow p_{0 d} \\
\sigma_{i} & \rightarrow \sigma_{d} \\
m_{i} & \rightarrow m_{d} \\
\xi_{i} & \rightarrow \xi_{d} .
\end{aligned}
$$

The formulation of the conditional cochannel interference probability for three different propagation conditions is described as follows.

1) Rayleigh Fading Only: In the event of incoherent cumulation with equal local mean power, i.e., $p_{0 i}=p_{0}$, the pdf for the joint interference power $p_{n}$ is obtained by convolving (4) $n$ times and given by gamma distribution [18], [19]

$$
f_{p_{n}}\left(p_{n}\right)=\frac{1}{p_{0}} \frac{\left(p_{n} / p_{0}\right)^{n-1}}{(n-1) !} \exp \left(-\frac{p_{n}}{p_{0}}\right) .
$$

The conditional cochannel interference can be derived using
TABLE I

Standard deviation $\left(\sigma_{u}\right)$ and Area Median Power $\left(m_{u}\right)$ FOR $n=1$ TO 6 INTERFERERS SUBJECT TO LOG-NORMAL Shadowing. Initial Value of $m_{i}=0 \mathrm{~dB}$

\begin{tabular}{ccccc} 
& \multicolumn{2}{c}{$\sigma_{i}=6 \mathrm{~dB}, \mathrm{~m}_{i}=0 \mathrm{~dB}$} & & \multicolumn{2}{c}{$\sigma_{i}=12 \mathrm{~dB}, m_{i}=0 \mathrm{~dB}$} \\
$n$ & $\sigma_{u}(\mathrm{~dB})$ & $m_{u}(\mathrm{~dB})$ & $\sigma_{u}(\mathrm{~dB})$ & \multicolumn{1}{c}{$m_{u}(\mathrm{~dB})$} \\
\hline 1 & 6.00000 & 0.00000 & 12.00000 & 0.00000 \\
2 & 4.57930 & 4.57589 & 9.58168 & 7.45252 \\
3 & 3.93286 & 6.90771 & 8.39718 & 11.20030 \\
4 & 3.53877 & 8.43359 & 7.65698 & 13.61733 \\
5 & 3.25742 & 9.56948 & 7.13423 & 15.37234 \\
6 & 3.03997 & 10.47850 & 6.73756 & 16.73845 \\
\hline
\end{tabular}

(2), (4), (6), and (7)

$$
\begin{aligned}
F(C I \mid n)=\int_{0}^{\infty} d p_{n} \int_{0}^{\alpha p_{n}} & \frac{1}{p_{0} p_{0 d}} \frac{\left(p_{n} / p_{0}\right)^{n-1}}{(n-1) !} \\
& \cdot \exp \left(-\frac{p_{d}}{p_{0 d}}-\frac{p_{n}}{p_{0}}\right) d p_{d} .
\end{aligned}
$$

After integrating (8) becomes

$$
F(C I \mid n)=1-\left(\frac{1}{\alpha p_{0} / p_{0 d}+1}\right)^{n} .
$$

2) Log-Normal Shadowing Only: The sum of $n$ stochastic independent log-normal variables can be well approximated by another log-normal variable [11], [12]. Schwartz and Yeh [12], [13] derived exact expressions for the mean and variance for the sum of two log-normal variables and then used a recursive approach to approximate the mean, $\xi_{u}$, and the variance, $\sigma_{u}^{2}$, for $n$ variables. Therefore, the pdf for $p_{0 u}\left(=\sum_{i=1}^{n} p_{0 i}\right)$ is of the form (5) with the following substitutions:

$$
\begin{aligned}
p_{0 i} & \rightarrow p_{0 u} \\
\sigma_{i} & \rightarrow \sigma_{u} \\
m_{i} & \rightarrow m_{u} \\
\xi_{i} & \rightarrow \xi_{u} .
\end{aligned}
$$

Substituting (6) and (10) in (2) yields the conditional cochannel interference probability

$$
\begin{aligned}
F(C I \mid n)= & \int_{0}^{\infty} d p_{0 u} \int_{0}^{\alpha p_{0 u}} \frac{1}{2 \pi \sigma_{d} \sigma_{u} p_{0 d} p_{0 u}} \\
& \cdot \exp \left\{-\left(\ln p_{0 u}-m_{u}\right)^{2} / 2 \sigma_{u}^{2}\right\} \\
& \cdot \exp \left\{-\left(\ln p_{0 d}-m_{d}\right)^{2} / 2 \sigma_{d}^{2}\right\} d p_{0 d}
\end{aligned}
$$

Equation (11) simplifies to

$$
F(C I \mid n)=\frac{1}{\sqrt{ }(2 \pi)} \int_{x}^{\infty} \exp \left(-t^{2} / 2\right) d t
$$

where

$$
x \triangleq\left[\ln \left(\xi_{d} / \alpha \xi_{u}\right)\right] /\left(\sigma_{d}^{2}+\sigma_{u}^{2}\right)^{1 / 2} .
$$

Table I gives values for $m_{u}$ and $\sigma_{u}$ as a function of number of interferers, $n$, with the initial value of $m_{i}=0 \mathrm{~dB}$.

3) Rayleigh Fading plus Log-Normal Shadowing: In the 
TABLE II

Standard Deviation $\left(\sigma_{n}\right)$ And Area Median Power $\left(m_{n}\right)$ FOR $n=1$ TO 6 INTERFERERS SUbJect TO RAYLeIGH FadING and Log-Normal Shadowing. Initial Value of $m_{i}=0 \mathrm{~dB}$

\begin{tabular}{rrrrr}
\hline & \multicolumn{2}{c}{$\sigma_{i}=6 \mathrm{~dB}, \mathrm{~m}_{i}=0 \mathrm{~dB}$} & \multicolumn{2}{c}{$\sigma_{i}=12 \mathrm{~dB}, m_{i}=0 \mathrm{~dB}$} \\
$n$ & $\sigma_{n}(\mathrm{~dB})$ & \multicolumn{1}{c}{$m_{n}(\mathrm{~dB})$} & $\sigma_{n}(\mathrm{~dB})$ & \multicolumn{1}{c}{$m_{n}(\mathrm{~dB})$} \\
\hline 1 & 7.00525 & -1.50515 & 12.53290 & -1.50515 \\
2 & 5.41069 & 3.51069 & 10.02766 & 6.22217 \\
3 & 4.66320 & 6.05705 & 8.79895 & 10.10659 \\
4 & 4.20419 & 7.71282 & 8.03063 & 12.61139 \\
5 & 3.87870 & 8.93309 & 7.48808 & 14.42947 \\
6 & 3.62925 & 9.89978 & 7.07652 & 15.84395 \\
\hline
\end{tabular}

general case of combined multipath fading and shadowing, considering the individual, stochastically independent signals add incoherently [19], the composite pdf for the (individual) $i$ th interfering signal power can be approximated by the $\log$-normal pdf with logarithmic variance $\left[\sigma_{i}^{2}+\ln (2)\right]$ and area median power $\xi_{i} / \sqrt{2}$ [16]. According to the suggestion of Schwartz and Yeh [12], the composite pdf for the (total) interference sum $p_{n}$ can then be approximated by a pure log-normal distribution with the appropriate moments

$$
f_{n}\left(p_{n}\right)=\frac{1}{\sqrt{(2 \pi) \sigma_{n} p_{n}}} \exp \left\{-\left(p_{u}-m_{n}\right)^{2} / 2 \sigma_{n}^{2}\right\} \text {. }
$$

Here $p_{u} \triangleq \ln p_{n}$, while $\xi_{n}\left(=\exp m_{n}\right)$ and $\sigma_{n}^{2}$ are the area median (interference) power and variance, respectively, of the equivalent log-normal variable. They are found in accordance with the procedure in [12], [13]. Hence, using (2)-(6) and (14) the conditional cochannel interference probability is given as

$$
\begin{aligned}
F(C I \mid n)= & \int_{0}^{\infty} d p_{0 d} \int_{0}^{\infty} d p_{n} \int_{0}^{\alpha p_{n}} \frac{1}{2 \pi \sigma_{d} \sigma_{n} p_{0 d}^{2} p_{n}} \\
& \cdot \exp \left(-\frac{p_{d}}{p_{0 d}}\right) \exp \left[\left\{-\left(\ln p_{n}-m_{n}\right)^{2} / 2 \sigma_{n}^{2}\right\}\right. \\
& \left.+\left\{-\left(\ln p_{0 d}-m_{d}\right)^{2} / 2 \sigma_{d}^{2}\right\}\right] d p_{d}
\end{aligned}
$$

After repeated integration, (15) becomes

$$
F(C I \mid n)=\frac{1}{\sqrt{ } \pi} \int_{-\infty}^{\infty} \exp \left(-w^{2}\right) f(w) d w
$$

where

$$
\begin{aligned}
f(w)= & 1-\exp \left[-\exp \left\{m_{n}+\alpha_{0}-m_{d}\right.\right. \\
& \left.\left.-\left[2\left(\sigma_{d}^{2}+\sigma_{n}^{2}\right)\right]^{1 / 2}\right\} w\right] \\
\alpha_{0} \triangleq & \ln \alpha .
\end{aligned}
$$

Table II gives values for $m_{n}$ and $\sigma_{n}$ as a function of the number of interferes, $n$, with the initial value of $m_{i}=0 \mathrm{~dB}$.

In order to compare the system performance in coherent and incoherent interference conditions, the conditional cochannel interference probability for the coherent case is also derived. To this end, we write the composite pdf for $n$ interfering signals with combined Rayleigh fading and log- normal shadowing for coherent addition using (3)-(6).

$$
\begin{aligned}
f_{f s}\left(p_{n}\right)=\frac{1}{\sqrt{ }(2 \pi) \sigma_{u}} \int_{0}^{\infty} \frac{1}{p_{0 u}^{2}} \exp \left(-p_{n} / p_{0 u}\right) \\
\cdot \exp \left\{-\left(\ln p_{0 u}-m_{u}\right\}^{2} / 2 \sigma_{u}^{2}\right\} d p_{0 u} .
\end{aligned}
$$

The composite pdf for the desired signal with Rayleigh fading and log-normal shadowing is again of the form (19), with the following substitutions:

$$
\begin{aligned}
p_{n} & \rightarrow p_{d} \\
p_{0 u} & \rightarrow p_{0 d} \\
\sigma_{u} & \rightarrow \sigma_{d} \\
m_{u} & \rightarrow m_{d} .
\end{aligned}
$$

The conditional cochannel interference probability is obtained using (2), (19), and (20)

$$
\begin{aligned}
F(C I \mid n)= & \frac{1}{2 \pi \sigma_{d} \sigma_{u}} \int_{0}^{\infty} d p_{0 d} \int_{0}^{\infty} d p_{0 u} \int_{0}^{\infty} d p_{n} \int_{0}^{\alpha p_{n}} \\
& \cdot \frac{1}{p_{0 u}^{2} p_{0 d}^{2}} \exp \left\{-\left(\frac{p_{d}}{p_{0 d}}+\frac{p_{n}}{p_{0 u}}\right)\right\} \\
& \cdot \exp \left[\left\{-\left(\ln p_{0 u}-m_{u}\right)^{2} / 2 \sigma_{u}^{2}\right\}\right. \\
& \left.+\left\{-\left(\ln p_{0 d}-m_{d}\right)^{2} / 2 \sigma_{d}^{2}\right\}\right] d p_{d}
\end{aligned}
$$

Equation (21) reduces to

$$
\begin{aligned}
& F(C I \mid n)=\frac{1}{\sqrt{ } \pi} \int_{-\infty}^{\infty}\left[\left\{\exp \left(-u^{2}\right)\right\} /\right. \\
& \left.\quad\left\{1+\left(\xi_{d} / \alpha \xi_{u}\right) \exp \left[-\left\{2\left(\sigma_{d}^{2}+\sigma_{u}^{2}\right)\right\}^{1 / 2} u\right]\right\}\right] d u
\end{aligned}
$$

It is assumed throughout that all cochannel interferers are statistically independent and identically distributed. If, moreover, only interfering signals from the nearest neighboring six cochannel cells are considered, and both the blocking probability $B$ and the number of channels $n_{c}$ are the same in all cells, $F_{n}(n)$ can be written as [3]

$$
F_{n}(n)=\left(\begin{array}{l}
6 \\
n
\end{array}\right) B^{n / n_{c}}\left(1-B^{1 / n_{c}}\right)^{6-n}, \quad n=0,1, \cdots, 6 .
$$

It has been noted that $F(n)$ can also be expressed in terms of carried traffic $a_{c}$ per channel [1], [2]

$$
F_{n}(n)=\left(\begin{array}{l}
6 \\
n
\end{array}\right) a_{c}^{n}\left(1-a_{c}\right)^{6-n}
$$

where $a_{c}=A_{c} / n_{c}$ and $A_{c}$ is the carried traffic per cell. Generally, (24) appears to be the more accepted representation in traffic analysis [20].

\section{Reuse Distance}

The (normalized) cochannel reuse distance, $R_{u}$, is defined as the ratio of the distance $D$ between the centers of the nearest neighboring cochannel cells and the cell radius $R$, 


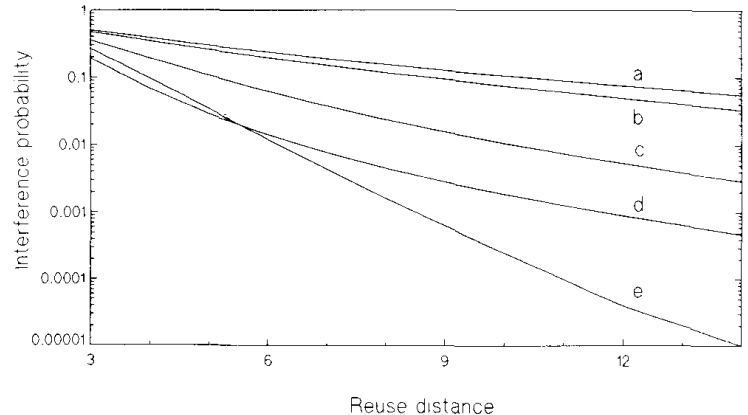

Fig. 1. Cochannel interference probability versus normalized reuse distance for protection ratio $\alpha=8 \mathrm{~dB}$, blocking probability $B=0.02$, carried traffic per cell $A_{c}=5$ erlang, and $n_{c}=10$ channels. Incoherent cumulation of interferers for: (a) combined Rayleigh fading and shadowing ( $\sigma=12$ $\mathrm{dB})$. (b) shadowing only ( $\sigma=12 \mathrm{~dB})$. (c) combined Rayleigh fading and shadowing $(\sigma=6 \mathrm{~dB})$. (d) Rayleigh fading only $(\sigma=0)$. (e) shadowing only $(\sigma=6 \mathrm{~dB})$.

i.e.,

$$
R_{u} \triangleq \frac{D}{R}
$$

The signal-to-interference ratio at a receiving base station located at the cell center is

$$
\frac{\xi_{d}}{\xi_{u}}=\frac{R^{-\gamma}}{\left.D^{-\gamma}\left[\exp \sum_{i=1}^{n} G_{l i}\right)\right]}
$$

where $\gamma$ is the ground-wave propagation path-loss slope (we take $\gamma=4$ ), $n$ is the number of cochannel interfering cells (here $n=6$ ), and $G_{l i}$ is a correction to the area median power which is dependent on $n$ and given by [13, eq. (8)]. Using (25) and (26), $R_{u}$ can be written as

$$
R_{u}=\left[\frac{\xi_{d}}{\xi_{u}} \exp \left(\sum_{i=1}^{n} G_{l i}\right)\right]^{1 / 4}
$$

Now, the cochannel interference probability can be computed as a function of $R_{u}$, for the three cases i), ii), and iii). The results are shown in Fig. 1 for $\sigma_{i}=\sigma_{d}=\sigma=6$ and $12 \mathrm{~dB}$. It is confirmed from Fig. 1 that a large reuse distance is essential to avoid excessive values of cochannel interference. It is also observed that shadowing is the predominant factor in determining the reuse distance and the cochannel interference probability, in particular for large $\sigma$.

Typical results for cochannel interference probabilities in the event of pure log-normal shadowing with $\sigma=6$ and 12 $\mathrm{dB}, \alpha=8 \mathrm{~dB}, n_{c}=10$, and $B=0.02$, are shown in Fig. 2, for both Fenton's classical method [11] and Schwartz and Yeh's technique [12], [13]. It is seen from Table III that Schwartz and Yeh's method results in up to $89 \%$ higher cochannel interference probabilities for $R_{u}=15$.

\section{SPECTRum EFficiency}

Spectrum efficiency, $E_{s}$, is defined as the carried traffic per cell, $A_{c}$, divided by the product of bandwidth per channel, $W$, number of channels per cell, $n_{c}$, and cluster

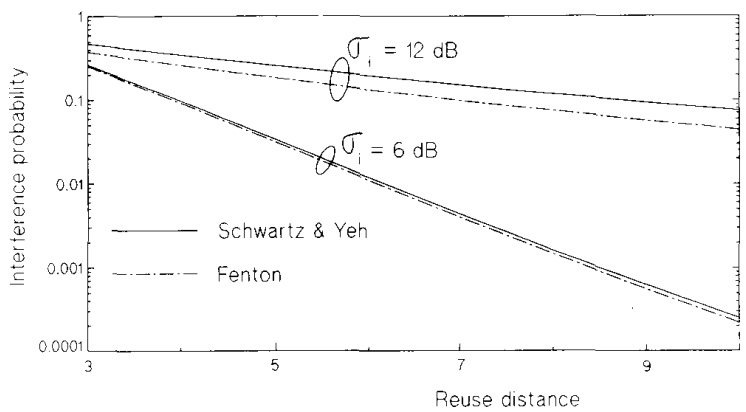

Fig. 2. Cochannel interference probability versus normalized reuse distance for $\alpha=8 \mathrm{~dB}, A_{c}=5$ erlang, $B=0.02$, and $n_{c}=10$ for shadowing only ( $\sigma=6$ and $12 \mathrm{~dB}$ ), calculated using Fenton's and Schwartz and Yeh's techniques.

TABLE III

Percentage Difference in Cochannel Interference Probability for Log-Normal Shadowing Environment Only, Comparing Schwartz and Yeh's Techniques with Fenton's Method FOR $\alpha=8 \mathrm{~dB}, n_{c}=10$ AND $B=0.02$

\begin{tabular}{rrc}
\hline$R_{u}$ & $\sigma_{i}=6 \mathrm{~dB}$ & $\sigma_{i}=12 \mathrm{~dB}$ \\
\hline 3 & $4 \%$ & $25 \%$ \\
6 & $9 \%$ & $47 \%$ \\
9 & $12 \%$ & $64 \%$ \\
12 & $14 \%$ & $78 \%$ \\
15 & $15 \%$ & $89 \%$ \\
\hline
\end{tabular}

size $C$ cells, given a unit cell area of $S$. Thus

$$
E_{s} \triangleq \frac{A_{c}}{n_{c} W C S} \text { erlang } / \mathrm{MHz} / \mathrm{km}^{2} .
$$

Carried traffic can be obtained by

$$
A_{c} \triangleq A(1-B)
$$

where $A$ is the offered traffic per cell in erlang. The blocking probability $B$ is determined using the Erlang- $B$ formula [20], [21]:

$$
B=\frac{A^{n} c}{n_{c} ! \sum_{n=0}^{n_{c}}\left(\frac{A^{n}}{n !}\right)} .
$$

The cells are assumed to form a cluster of size $C$, located around a reference cell and repeated around each of its cochannel cells. However, the exact shape of a valid cluster need not be precisely specified [17]. The cluster size is taken on the form

$$
C=i^{2}+i j+j^{2}, \quad i, j \geq 0
$$

with integer $i$ and $j$.

The reuse distance and the number of cells per cluster are related by [17], [23]

$$
R_{u}=(3 C)^{1 / 2} .
$$

Fig. 3 shows plots for the cluster size and reuse distance determined using (32) and for spectrum efficiency versus reuse distance obtained using (28) and (29), taking $W=25$ $\mathrm{kHz}, B=0.02, A_{c}=5$ erlang, $n_{c}=10$, and $S=1 \mathrm{~km}^{2}$. 


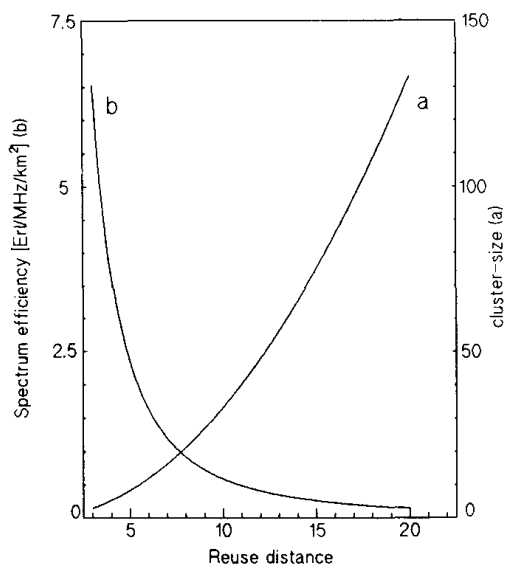

Fig. 3. Influence of normalized reuse distance $\left(R_{u}\right)$. (a) cluster size $(C)$. (b) spectrum efficiency $\left(E_{s}\right)$ for $A_{c}=5$ erlang, $B=0.02, W=25 \mathrm{kHz}$, $S=1 \mathrm{~km}^{2}$, and $n_{\mathrm{c}}=10$.

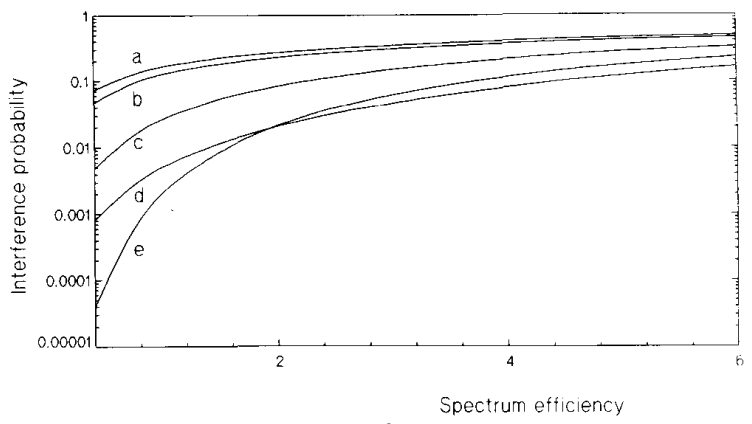

Fig. 4. Cochannel interference probability versus spectrum efficiency $\left(E_{s}\right)$. channel bandwidth $W=25 \mathrm{kHz}$; unit cell area $S=1 \mathrm{~km}^{2}$; other parameters as in Fig. 1.

It can be inferred from Figs. 1 and 3 that the spectrum efficiency can be increased by accepting an increase in the cochannel interference probability; this is seen directly from Fig. 4. The cluster size is seen to play an important role in determining the optimum spectrum efficiency for meeting a certain system requirement: a decrease in cluster size (and hence reuse distance) increases the spectrum efficiency. Referring to Fig. 1, this is equivalent to accepting a higher cochannel interference probability. This conclusion can also be drawn from Fig. 4, which shows the relation between interference probability and spectrum efficiency.

To ascertain the difference between coherent and incoherent cumulation of interference, Table IV compares the corresponding cochannel interference probabilities for $\sigma=6$ and $12 \mathrm{~dB}$, and different reuse distances. While coherent conditions do allow smaller interference probability, Table IV shows that the difference is rather small (below $10 \%$ in the cases considered), and decreases at larger reuse distances. In the following numerical examples, we, therefore, confine ourselves to incoherent cumulation interferes.

Figs. 5 and 6 show interference probability as a function of spectrum efficiency, with protection ratio (Fig. 5) and the carried traffic (Fig. 6) as a parameter. Clearly, the spectrum efficiency can be increased by tolerating a lower value of

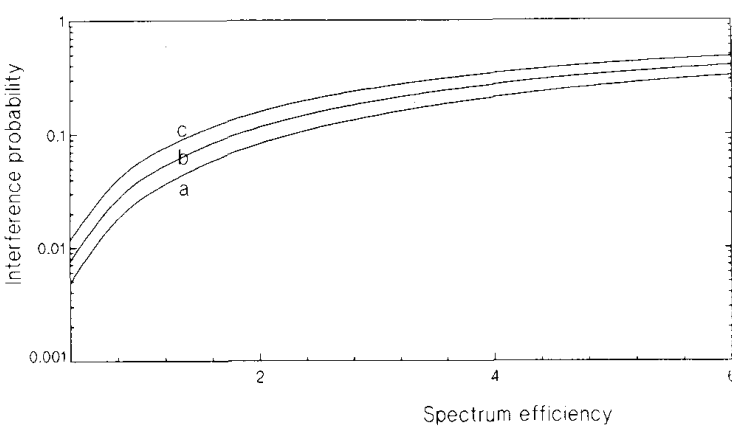

Fig. 5. Cochannel interference probability versus spectrum efficiency for combined Rayleigh fading and shadowing $(\sigma=6 \mathrm{~dB})$ using incoherent cumulation of interference with $A_{c}=5$ erlang, $B=0.02, n_{c}=10, S=1$ $\mathrm{km}^{2}, W=25 \mathrm{kHz}$, and (a) $\alpha=8 \mathrm{~dB}$, (b) $\alpha=10 \mathrm{~dB}$, (c) $\alpha=12 \mathrm{~dB}$.

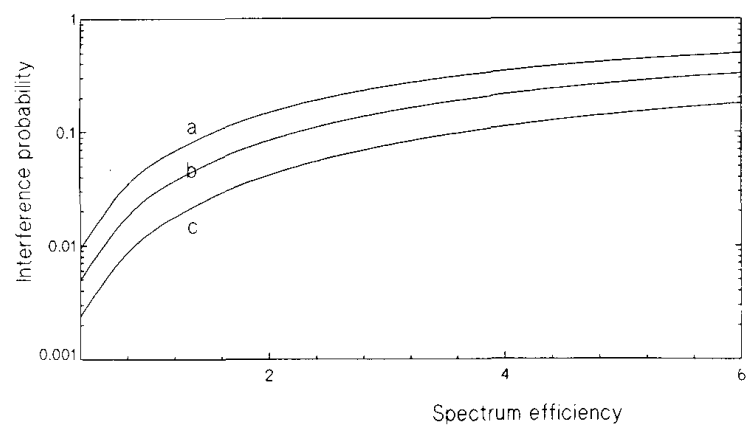

Fig. 6. Cochannel interference probability versus spectrum efficiency for combined Rayleigh fading and shadowing using incoherent cumulation of interference with $n_{c}=10, S=1 \mathrm{~km}^{2}, \sigma=6 \mathrm{~dB}, W=25 \mathrm{kHz}, \alpha=8 \mathrm{~dB}$, (a) $a_{c}=0.99$ erlang/channel ( $B=0.8$ ), (b) $a_{c}=0.5$ erlang $/$ channel $(B=$ $0.02)$, (c) $a_{c}=0.23$ erlang $/$ channel $(B=0.0001)$.

TABLE IV

Cochannel Interference Probability for the Coherent AND INCOHERENT CUMULATION OF COCHANNEL INTERFERERS With $\sigma=6$ AND $12 \mathrm{~dB}$ as a Function of Reuse Distance

\begin{tabular}{ccccc}
\hline & \multicolumn{2}{c}{$\sigma_{i}=6 \mathrm{~dB}$} & \multicolumn{2}{c}{$\sigma_{i}=12 \mathrm{~dB}$} \\
$R_{u}$ & incoherent & coherent & incoherent & coherent \\
\hline 3 & 0.35867 & 0.32317 & 0.50531 & 0.47402 \\
4 & 0.19453 & 0.17840 & 0.38644 & 0.36220 \\
5 & 0.10791 & 0.10107 & 0.30083 & 0.28155 \\
6 & 0.06236 & 0.05954 & 0.23971 & 0.22285 \\
7 & 0.03767 & 0.03655 & 0.19187 & 0.17910 \\
8 & 0.02374 & 0.02333 & 0.15501 & 0.14574 \\
9 & 0.01555 & 0.01543 & 0.12743 & 0.11995 \\
\hline
\end{tabular}

protection ratio (Fig. 5). More surprising on first sight is the increase of spectrum efficiency obtained with a lower carried traffic $A_{c}$, given a fixed interference probability (Fig. 6). This is caused by the much stronger impact in (28) of the corresponding reduction in the necessary reuse distance $R_{u}$ (32). Thus a higher blocking probability does not necessarily result in higher spectrum efficiency.

Figs. 7-9 compare the spectral efficiencies of the different modulation methods of interest in cellular telephony shown in Table $\mathrm{V}$. The protection ratio $(\alpha)$ is defined as the minimum value of the wanted (carrier)-to-unwanted (interference) signal ratio at the receiver input such that a specified reception 


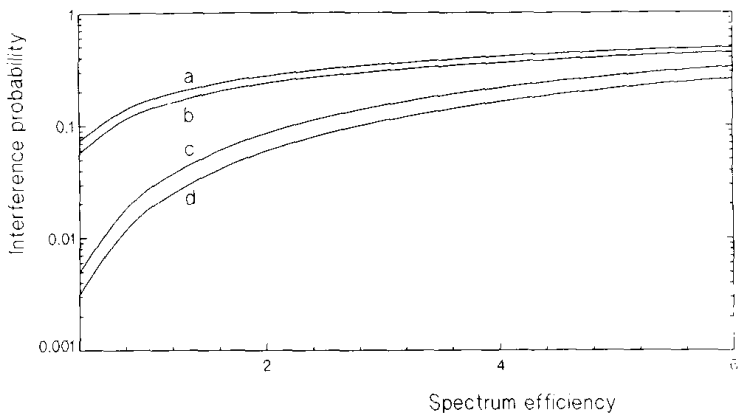

Fig. 7. Cochannel interference probability versus spectrum efficiency for $a_{\mathrm{c}}=0.5 \mathrm{erlang} / \mathrm{channel}, \boldsymbol{B}=0.02, S=1 \mathrm{~km}^{2}$, and $n_{c}=10$ for combined fading and shadowing using incoherent cumulation of interference with (a) $25-\mathrm{kHz}$ digital modulation with 8-dB protection ratio and $\sigma=12 \mathrm{~dB}$, (b) $12.5-\mathrm{kHz}$ FM with $12-\mathrm{dB}$ protection ratio and $\sigma=12 \mathrm{~dB}$, (c) $25-\mathrm{kHz}$ digita modulation with 8-dB protection ratio and $\sigma=6 \mathrm{~dB}$, (d) $12.5-\mathrm{kHz}$ FM with 12-dB protection ratio and $\sigma=6 \mathrm{~dB}$.

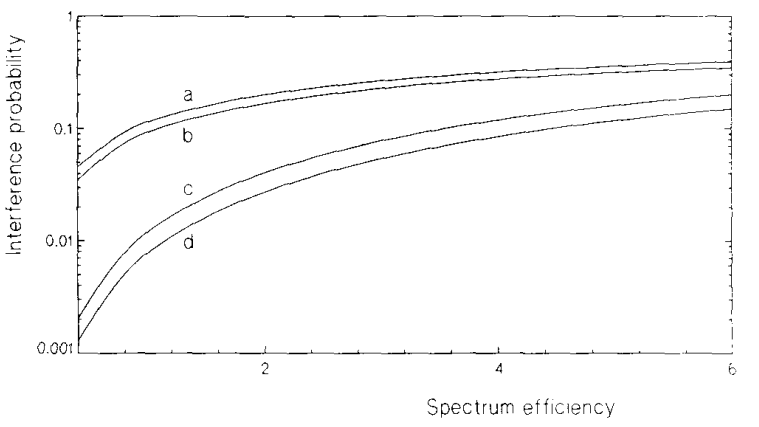

Fig. 8. Cochannel interference probability versus spectrum efficiency for $a_{c}=0.5$ erlang/channel, $B=0.02$, and $n_{c}=10$ for combined fading and shadowing using incoherent cumulation of interference with (a) $5-\mathrm{kHz}$ SSB with $\alpha=18 \mathrm{~dB}$ and $\sigma=12 \mathrm{~dB}$, (b) $5-\mathrm{kHz}$ SSB with $\alpha=16 \mathrm{~dB}$ and $\sigma=12 \mathrm{~dB}$, (c) $5-\mathrm{kHz}$ SSB with $\alpha=18 \mathrm{~dB}$ and $\sigma=6 \mathrm{~dB}$, (d) $5-\mathrm{kHz}$ SSB with $\alpha=16 \mathrm{~dB}$ and $\sigma=6 \mathrm{~dB}$.

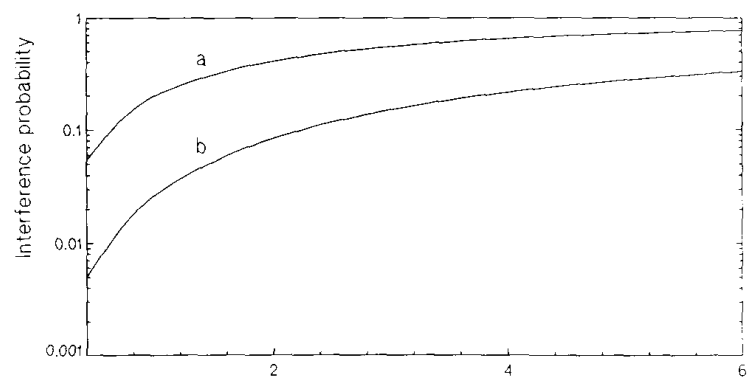

Spectrum efficiency

Fig. 9. Cochannel interference probability versus spectrum efficiency for $a_{c}=0.5 \mathrm{erlang} / \mathrm{channel}, B=0.02$, and $n_{c}=10$ for combined fading and shadowing using incoherent cumulation of interference with (a) $30-\mathrm{kHz} F M$ with $18 \mathrm{~dB}$ protection radio and $\sigma=6 \mathrm{~dB}$, (b) $25-\mathrm{kHz}$ digital modulation with $8 \mathrm{~dB}$ protection ratio and $\sigma=6 \mathrm{~dB}$.

quality of the wanted signal is achieved at the receiver output [27]. For analog FM systems, as used for the U.S. Advanced Mobile Phone Services (AMPS) system and the U.K. Total Access Communication System (TACS), satisfactory operation requires approximately $\alpha=18 \mathrm{~dB}$ [17], [25]-[28]. The channel spacing in AMPS is $30 \mathrm{kHz}$, whereas in TACS a closer channel spacing of $25 \mathrm{kHz}$ has been adopted. The
TABLE V

Channel Spacing and Protection Ratio for Different Modulation METHOdS

\begin{tabular}{lccl}
\hline $\begin{array}{c}\text { Modulation } \\
\text { method }\end{array}$ & $\begin{array}{c}\text { Channel } \\
\text { spacing }\end{array}$ & $\begin{array}{c}\alpha(\mathrm{dB}) \\
\text { Protection } \\
\text { ratio }\end{array}$ & \multicolumn{1}{c}{ References } \\
\hline Analog FM & $12.5 \mathrm{kHz}$ & 12 & {$[8],[24]$} \\
Analog FM & $30 \mathrm{kHz}$ & 18 & {$[17],[25]-[27]$} \\
Digital modulation & $25 \mathrm{kHz}$ & 8 & {$[25]$} \\
SSB & $5 \mathrm{kHz}$ & 16 and 18 & {$[8],[24],[27]$} \\
\hline
\end{tabular}

Nordic Mobile Telephone (NMT-900) system even uses a $12.5-\mathrm{kHz}$ channel spacing. In the future Pan-European digitally modulated system Groupe Speciale Mobile (GSM), $\alpha$ will be approximately $9.5 \mathrm{~dB}$ [25]; here, we have used $8 \mathrm{~dB}$ and a channel spacing of $25 \mathrm{kHz}$ (an European standard) for the purpose of computations. For the sake of completeness, the spectrum efficiency is also evaluated (Fig. 8) for SSB. An SSB system with a $5-\mathrm{kHz}$ channel spacing suffers similar subjective effects of cochannel interference as a $25 \mathrm{kHz}$ FM system under real mobile radio field conditions [27]. In both cases, a signal-to-interference ratio of the order of $16 \mathrm{~dB}$ is necessary to achieve a fair quality of reception. Therefore, the efficiency of SSB systems is evaluated for $\alpha=16$ and 18 $\mathrm{dB}$ with a $5-\mathrm{kHz}$ channel spacing.

The computational results (Figs. 7-9) show that SSB offers the highest efficiency among the modulation techniques considered in this paper. Our efficiency ranking of these methods agree with [8] but also include for $30-\mathrm{kHz}$ spaced FM (AMPS) and the digital GSM.

\section{SYSTEM BandwidTH}

System bandwidth represents the total bandwidth required to serve a cluster. Thus it can be expressed as the product of the number of channels per cluster and the bandwidth per channel, i.e.,

$$
S_{w} \triangleq n_{c} W C=\frac{A_{c}}{S E_{s}} \mathrm{MHz} .
$$

Thus as distinct from the spectrum efficiency, the system bandwidth is an absolute measure of the frequency resources required to meet a certain traffic demand in a given area. The cluster size $C$ and the modulation method influence the system complexity and, hence, the economic resources required to realize the cellular network and the mobile terminals. Thus a judicious trade-off between system options can be based on $S_{w}, C$ and the modulation parameters.

To illustrate the typical differences between cellular system options, Table VI shows spectrum efficiency, system bandwidth and cluster size for the two FM systems, one digital modulation system (DM) and two SSB systems dealt with in Figs. 7-9. The cluster size $C$ is approximated by the nearest valid number, according to (31). The Table clearly reveals the relative merits of the different systems, in terms of frequency demands and cellular system complexity.

\section{Conclusions}

Cochannel interference probabilities for mobile radio systems exposed to realistic propagation impairments have been 
TABLE VI

Spectrum Efficiency $E_{S}$, System Bandwidth $S_{w}$ and Cluster

Size $C$ for Different Types of Modulation Technigues with $n_{c}=10, B=0.02, a_{c}=0.5$ ERLANG $/$ CHANNEL, AND $F(C I \mid n)=10^{-1}$ in a Combined Fading and Shadowing Environment WITH $\sigma=6 \mathrm{~dB}$

\begin{tabular}{cccr}
\hline $\begin{array}{c}\text { Type of } \\
\text { modulation }\end{array}$ & $E_{s}\left(\right.$ erlang $\left./ \mathrm{MHz} / \mathrm{km}^{2}\right)$ & $S_{w}(\mathrm{MHz})$ & $C$ \\
\hline $\mathrm{FM}_{30}$ & 0.6 & 8.4 & 28 \\
$\mathrm{DM}_{25}$ & 2.2 & 2.25 & 9 \\
$\mathrm{FM}_{12.5}$ & 2.8 & 2 & 16 \\
$\mathrm{SSB}_{18}$ & 3.6 & 1.4 & 28 \\
$\mathrm{SSB}_{16}$ & 4.5 & 1.25 & 25 \\
\hline
\end{tabular}

calculated using Fenton's classical method and compared with our values, obtained by Schwartz and Yeh's technique. Our results indicate significantly higher interference probabilities, up to $89 \%$ more than suggested by the classica! method. This indicates that earlier results for cochannel interference calculations in cellular radio systems based on Fenton's method are optimistic. The use of Schwartz and Yeh's technique is recommended for mobile scenarios in which the variance of the shadowing lies between 6 and $12 \mathrm{~dB}$.

Earlier studies of cochannel interference probability in a cellular mobile network were carried out assuming coherent cumulation of interference. Now, results are also available for the more realistic assumption of incoherent cumulation of interfering signals with independent Rayleigh fading, lognormal shadowing and UHF ground-wave path loss. Computed results show that coherent cumulation of interferers leads to somewhat lower values of cochannel interference probability, i.e., a slightly higher spectrum efficiency than with incoherent cumulation. However, for large reuse distances the differences become minute.

A detailed assessment of the spectrum efficiency and system bandwidth of general cellular systems has been given. It is confirmed that the spectrum efficiency is higher for lower protection ratio, and that a tradeoff has to be made between cluster size and cochannel interference probability to achieve the desired spectrum efficiency. In particular, it cannot be assumed that higher carried traffic (higher blocking probability) always leads to better spectral efficiencies, because of the associated increase in cluster size required to maintain a given interference probability.

In practical terms, a design trade-off will more often be between the overall system bandwidth (absolute bandwidth required to provide a certain service) and the associated network complexity (in terms of cluster size, modulation type, etc.). The approach described in this paper would assist planners and designers in obtaining a more realistic assessment of different system options, especially in the many cases where the shadowing of mobiles cannot be assumed to be moderate.

\section{ACKNOWLEDGMENT}

The authors are grateful to Prof. Dr. Jens Arnbak and to Ir. Wim van Blitterswijk, PTT Research, The Netherlands, for discussions. They would like to thank the anonymous reviewers for valuable suggestions, and Nel Kay-de Jong for preparing the manuscript.

\section{REFERENCES}

[1] Y. Nagata and Y. Akaiwa, "Analysis for spectrum efficiency in single cell trunked and cellular mobile radio," IEEE Trans. Veh. Technol. vol. VT-35, pp. 100-113, Aug. 1987.

[2] $K$. Daikoku and $H$. Ohdate, "Optimal channel reuse in cellular land mobile radio systems," IEEE Trans. Veh. Technol., vol. VT-32, pp. 217-224, Aug. 1983.

[3] R. Muammar and S. C. Gupta, "Cochannel interference in highcapacity mobile radio systems," IEEE Trans. Commun., vol. COM-30, pp. 1973-1978, Aug. 1982.

[4] D. C. Cox, "Cochannel interference considerations in frequency reuse small-coverage-area radio systems," IEEE Trans. Commun., vol. COM-30, pp. 135-142, Jan. 1982

[5] M. Hata, K. Kinoshita, and K. Hirade, "Radio link design of cellular land mobile communication systems," IEEE Trans. Veh. Technol., vol. VT-31, pp. 25-31, Jan. 1982.

[6] L. Jasinski, "Statistical analysis of communications range and reliability in the presence of interference," IEEE Trans. Veh. Technol., vol. VT-30, pp. 123-130, Aug. 1981.

[7] W. Gosling, "A simple mathematical model of co-channel and adjacent channel interference in land mobile radio," IEEE Trans. Veh. Technol., vol. VT-29, pp. 361-364, Nov. 1980.

[8] R. C. French, "The effect of fading and shadowing on co-channel reuse in mobile radio," IEEE Trans. Veh. Technol., vol. VT-28, pp. 171-181, Aug. 1979.

[9] F. Hansen and F. Meno, "Mobile fading-Rayleigh and lognormal superimposed," IEEE Trans. Veh. Technol., vol. VT-26, pp. 332-335, Nov. 1977

[10] J. Stjernvall, "Calculation of Capacity and co-channel interference in a cellular system," in Proc. Nordic Seminar on Digital Land Mobile Radiocommunication, Espoo, Finland, Feb. 1988, pp. 209-217.

[11] L. F. Fenton, "The sum of log-normal probability distributions in scatter transmission systems," IRE Trans. Commun. Syst., vol. CS-8, pp. 57-67, Mar. 1960

[12] S. C. Schwartz and Y. S. Yeh, "On the distribution function and moments of power sums with log-normal components," Bell Syst. Tech. J., vol. 61, pp. 1441-1462, Sept. 1982.

[13] R. Prasad and J. C. Arnbak, "Comments on analysis for spectrum efficiency in single cell trunked and cellular mobile radio," IEEE Trans. Veh. Technol., vol. 37, pp. 220-222, Nov. 1988.

[14] _-, "Enhanced packet throughput in radio channels with shadowing," Electron. Lett., vol. 24, pp. 986-988, Aug. 1988.

[15] -, "Enhanced packet throughput in radio channels with fading and shadowing," in Proc. Canadian Conf. in Electrical and Computer Engineering, Vancouver, Canada, Nov. 1988, pp. 78-80.

[16] _ - "Effects of Rayleigh fading on packet radio channels with shadowing," in Proc. IEEE TENCON'89 Conf., Bombay, India, Nov. 1989 , pp. $546-548$.

[17] V. H. MacDonald, "The cellular concept," Bell Syst. Tech. J., vol. 58, pp. 15-41, Jan. 1979

[18] P. Beckman, Probability in Communication Engineering. New York: Harcourt, Brace and World, 1967.

[19] J. C. Arnbak and W. van Blitterswijk, "Capacity of slotted ALOHA in Rayleigh fading channels," IEEE J. Select. Areas Commun. vol. SAC-5, pp. 261-269, Feb. 1987.

[20] J. A. Elldin and G. Lind, Elementary Telephone Traffic Theory. Telefonaktiebolaget LM ERICSSON, Stockholm, Sweden, 1967.

[21] M. Schwartz, Telecommunication Networks Protocols, Modeling and Analysis. Reading, MA: Addison Wesley, 1987. 
[22] J. J. Bellamy, Digital Telephony. New York: Wiley, 1982

[23] W. C. Y. Lee, "Elements of cellular mobile radio systems," IEEE Trans. Veh. Technol., vol. VT-35, pp. 48-56, May 1986.

[24] W. Gosling, "Protection ratio and economy of spectrum use in land mobile radio," Proc. Inst. Elect. Eng., vol. 127, pt. F, pp. 174-178, June 1980.

[25] J. Walker, Mobile Information Systems. Boston, MA: Artech House, 1990

[26] W. C. Y. Lee, Mobile Cellular Telecommunications Systems. New York: McGraw-Hill, 1989.

[27] Recommendations and Reports of the CCIR, Vol. VIII-I, 1986.

[28] C. J. Hughes and M. S. Appleby, "Definition of a cellular mobile radio system," Proc. Inst. Elect. Eng., vol. 132, pt. F, pp. 416-424, Aug. 1985

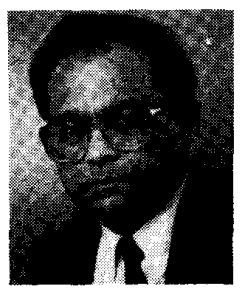

Ramjee Prasad (M'88-SM'90) was born in India on July 1,1946 . He received the B.Sc. (Engg.) degree from Bihar Institute of Technology, Sindri, India, in 1968, and the M.Sc. (Engg.) and Ph.D. degrees from Birla Institute of Technology (BIT), Ranchi, India, in 1970 and 1979, respectively.

He joined BIT as Senior Research Fellow in 1970 and became Associate Professor in 1980. During 1983-1988 he was with the University of Dar es Salaam (UDSM), Tanzania, where he became Professor in Telecommunications at the Department of Electrical Engineering in 1986. Since February 1988, he has been with the Telecommunications and Traffic Control Systems Group, Delft University of Technology, The Netherlands, where he is actively involved in the area of mobile communications. While he was with BIT, he supervised many research projects in the area of microwave and plasma engineering. He was project responsible at UDSM for the collaborative project "Satellite Communications for Rural Zones" with Eindhoven University of Technology, The Netherlands. He has published over 70 technical papers. His current research interest lies in mobile communications, indoor wireless communications, and telematics.

Dr. Prasad is a Fellow of the Institution of Electronics and Telecommunication Engineers, India.

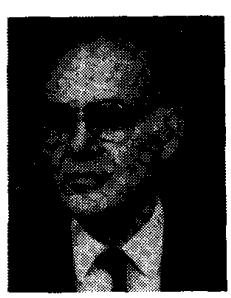

Adriaan Kegel was born in The Netherlands on November 4, 1932. He received the M.Sc. degree from Delft University, The Netherlands, in 1972.

He joined Philips in 1954 where he worked on the development of microwave link equipment as a Radio Engineer. In 1964 he joined Delft University of Technology. In 1970 he became Chairman of the "Working Group Indonesia," which developed the so-called "Electronic Blackboard." For this development the working group received an Award from the Scientific Radio Foundation Veder. After completion of the Indonesian Project in 1974, he continued his research work on the coding and transmission of graphical information. This work led to the development of several systems (e.g., Vidibord) and a number of publications. His recent interest is in research and education on mobile communication systems.

Mr. Kegel is a member of The Netherlands Electronics and Radio Society and the Federation of Telecommunication Engineers of the European Community. 